\title{
Accuracy Analysis of the Brownian Motion Approach for the Ballistic Resistance Estimation : Comparison of Numerical and Experimental Distributions
}

\author{
B. Tahenti $^{\mathrm{a}}$, F. Coghe ${ }^{\mathrm{b}}$, R. Nasri ${ }^{\mathrm{c}}$ \\ ${ }^{a}$ Military Academy of Fondouk Djedid, (Tunisia) \\ ${ }^{b}$ Royal Military Academy, (Belgium) \\ ${ }^{c}$ National Engineering School of Tunis, (Tunisia) \\ Email:tahenti@hotmail.com
}

\begin{abstract}
The ballistic resistance assessment is an active research field that deals with the estimation of the perforation probability of a given protection/bullet combination. The modelling challenge is to increase accuracy and precision of estimates based on a small sample of hidden penetration processes (limitation of experimental measurement tools due to the complexity of dynamic impact phenomenon). Actually, existing methods make use of the impacting bullet initial velocity and the protection response coded in a binary outcome ( 0 if perforation takes place and 1 if not). Recently, a Brownian motion approach has been proposed using the numerical integration of stochastic differential equations. This contribution analysis the sensitivity of the ballistic resistance estimations regarding the model parameters. To fulfil the desired goal, the numerical and experimental distribution of the estimates are compared. Available database allows the computation of a point estimation of the perforation probability at a given impact velocity. Statistical Bootstrap is performed to obtain the experimental distribution of the perforation probability in order to avoid costly experiments. In the other side, the model parameters uncertainty is propagated to the model estimations using Monte Carlo simulations. The resulted numerical distribution encompass the inherent stochastic model randomness and the model parameters uncertainty effect. Furthermore, the numerical experiments are generated as the experimental observations in the interest of a fair comparison. First, the numerical and experimental distributions are compared regarding the optimum values of the model parameters and randomly selected values over the tolerance intervals. It is concluded that the model presents a low sensitivity to the parameters estimation. Next, the experimental and numerical variance of the perforation probabilities are investigated. It is observed that the model estimations are in good agreement with the experimental one. Finally, the distribution of the mean difference between the numerical and experimental distributions is analysed as a function of the bullet impact velocity. Again, the model appears to have a low sensitivity to its parameters estimation uncertainty. Further-more, it is showed that the model performance depends on the bullet initial impact velocity. Therefore, it is suggested to further investigate the functional form of the stochastic differential equation coefficient in order to better estimate the perforation probability as a function of the initial impact velocity.
\end{abstract}

Keywords: Stochastic model, Brownian motion, ballistic resistance, model sensitivity, perforation probability 
B. Tahenti et al., Accuracy Analysis of the Brownian Motion Approach for the Ballistic Resistance...

\section{INTRODUCTION}

The ballistic resistance assessment of protection materials is an important issue either for manufacturer or purchaser. Therefore, a growing interest is addressed to the accuracy in the evaluation of materials resistance to ballistic threats. This may be of increasing concern especially in light of the constraining requirements on minimizing protection weight and increasing comfort. Actually, two key measurements are used in the ballistic evaluation of armour systems. The first consists in the ballistic limit velocity $V_{50}$, bullet impact velocity at which partial and complete penetration are equally likely. The second is the limit velocity $V_{0}$ which is attended to quantify the maximum impact velocity with zero perforation of the target among all the observed trials. The STANAG 2920 (NATO (2003)) defines a standardized method for the estimation of the $V_{50}$ velocity. Comparing different threat/protection combination using only the information provided by the $V_{50}$ velocity can be too restrictive. Recently, considerable attention has been devoted to the determination of the probability of perforation curve as a function of the impact velocities. For this reason, the focus of recent research has been the estimation of the material response variability under impact loading. Langlie (1963), Kneubuehl (2003) and Finney and Tattersfield (1952) proposed different purely statistical models. They assumed that the velocities with observed perforation of the target are normally distributed $V \sim N\left(V_{50}, \hat{\sigma}^{2}\right)$. Thus, the system response variability is described by the estimated standard deviation $\hat{\sigma}$. However, an emerging stochastic model based on the Brownian motion approach has been advanced by Coghe et al. (2016). Later, Tahenti et al. (2017) implemented this modelling idea using the Chi-square and Kolmogorov-Smirnov goodness of fit tests. The aim of this paper is the analysis of the accuracy of the Brownian motion approach in estimating experimentally computed probability of perforation. For this, statistical Bootstrap is performed to establish the distributions of the experimental estimations. In the other side, the numerical distributions are obtained by running Monte Carlo error propagation on the final results of Tahenti et al. (2017) Work. To fulfil the specified goal, the needed experimental database is introduced. Next, the stochastic model is briefly explained. In addition, statistical bootstrap and Monte Carlo error propagation concepts are developed in order to be applied in the present analysis. Finally, the retrieved results are discussed.

\section{EXPERIMENTAL AND NUMERICAL ESTIMATION}

Ballistic experiments are performed to observe the response of the protection subjected to the impact of a specific bullet with a given impact velocity. Then, the provided database is manipulated by existing models in various ways for the sake of estimating the $V_{50}$ and $\hat{\sigma}$ (for the Langlie (1963), Kneubuehl (2003) and Finney and Tattersfield (1952) models). In the current section, the experimental method and database needed for the estimation of the ballistic resistance are introduced. Furthermore, the emerging Brownian motion approach is summarized.

\subsection{Experimental Approach}

A conventional testing set-up is proposed by different standards such as the STANAG 2920 (NATO (2003)), the MIL-662F (Department of Defense (1997)) and the NIJ 0101.06 (National Institute of Justice (2008)). The recorded measurements are the bullet impact velocity $V_{i}$ and the protection reaction coded in a binary outcome; $U_{i}$ equal to zero if perforation takes place and 1 if not. Maldague et al. (2010) created a large database of impact

Table 1. Experimental results for the $5.56 \mathrm{~mm}$ projectile impacts against mild steel plates Maldague et al. (2010).

\begin{tabular}{|c|c|c|c|c|c|}
\hline Class $\mathbf{N r}$ & $\mathbf{V}_{\mathbf{m i n}}\left(\mathbf{m} \cdot \mathbf{s}^{\mathbf{- 1}}\right)$ & $\mathbf{V}_{\mathbf{m a x}}\left(\mathbf{m} \cdot \mathbf{s}^{-\mathbf{1}}\right)$ & Perforations & $\mathbf{I m p a c t s}$ & $\mathbf{F}_{\mathbf{e x p}}$ \\
\hline \hline 1 & 480 & 490 & 0 & 22 & 0.0 \\
\hline 2 & 490 & 500 & 2 & 63 & 0.0317 \\
\hline 3 & 500 & 510 & 27 & 109 & 0.2477 \\
\hline 4 & 510 & 520 & 48 & 82 & 0.5854 \\
\hline 5 & 520 & 530 & 130 & 157 & 0.8280 \\
\hline 6 & 530 & 540 & 67 & 73 & 0.9178 \\
\hline 7 & 540 & 550 & 28 & 28 & 1.0 \\
\hline
\end{tabular}


loading of a metallic plate. A two spaced mild steel plates were tested against the $5.56 \times 45 \mathrm{~mm}$ bullet. The total thickness of the protection is equal to $H=5.60 \mathrm{~mm}$. Table 1 recapitulates the collected experimental results. Statistically speaking, for each impacting velocity, the test should be repeated $\mathrm{n}$ times at the same condition. Therefore, an estimation of the experimental frequency of perforation can be performed. Given the complexity of controlling precisely the impact velocity, ballistic testing is conducted at various impact velocities. Finally, the experimental frequency $F_{\text {exp }}$ (where i=1,2...7 is referring to the corresponding class of velocities) is computed based on the number of perforation occurrence within classes of impact velocity as displayed in table 1 .

\subsection{Brownian Motion Approach}

In a recent paper by Tahenti et al. (2017), The bullet motion within the target has been modelled as a stochastic motion. In fact, the deterministic Newton's second law is transformed to a stochastic differential equation using the Brownian motion process. So, the system of equations that describes the random bullet motion is as follows :

$$
\left\{\begin{array}{l}
d V(t)=a(t, V) d t+\sigma(t, V) d W(t) \\
d X(t)=V(t) d t \\
X(0)=0 \text { and } V(0)=V_{i}
\end{array}\right.
$$

where $a(t, V)$ is the bullet deceleration, $V(t)$ is the instantaneous bullet velocity, $X(t)$ is the bullet position and $\sigma(t, V)$ is the diffusion coefficient. The Brownian motion $W(t)$ is a Gaussian process characterized by continuous paths and independent increments. The reader interested in the mathematical background and the numerical implementation of this modelling approach may be referred to Tahenti et al. (2017). Under the assumption of constant bullet deceleration and diffusion coefficient, Tahenti et al. (2017) validated his modelling approach with experimental observations using the parameters $\left(a=2.358 \times 10^{7} \mathrm{~m} \mathrm{~s}^{-2}, \sigma=4205\right)$ that are the best-found fit over three hundred optimization of the Chi-square and Kolmogorov-Smirnov goodness of fit criterion (the collected data $(a, \sigma)$ from all the applied optimization are called hereafter the optimization sample). The goal in this work is to compare the distribution of experimentally computed probability with these established based on the Brownian motion approach. In the next section statistical bootstrap and error propagation tools are brought to bear on the problem of comparing the probability distribution of experimental and numerical estimation.

\section{ERROR PROPAGATION}

\subsection{Statistical Bootstrap}

At a given impact velocity $V_{i}$ (or $\mathrm{i}^{\text {th }}$ class of velocities), the number of occurred perforations $\left(n_{i_{U_{i}=0}}\right.$ ) follows a Binomial law $B\left(n_{i}, F_{\text {exp }}\right)$ where $n_{i}$ is the total number of impacts in the considered class. The experimentally established contingency table provide a punctual estimation of the frequency of perforations as is displayed in table 1: the sample estimation $F_{\text {exp }}$. However, an empirical distribution of $F_{\text {exp }}$ can be approximated using statistical non-parametric bootstrap. Figure 1 shows a conceptual design of the bootstrapping technique. Namely, original observations are sampled with replacement using Monte Carlo simulations. Therefore, $\mathrm{N}$ simulated samples with equal size to the initial one are provided. Computing the expected values of the probability of perforation $\hat{F}_{\text {exp }}$ based on the new samples allow the construction of the bootstrap distribution per class of impact velocities i. It is to be noted that, in the present analysis, the bootstrap distribution is referred to as the experimental one given it was established on experimental observations.

\subsection{Uncertainty Propagation}

Tahenti et al. (2017) showed that the numerical model passes the Chi-square and Kolmogorov-Smirnov goodness of fit tests to experimental results for $\left(a \in\left[2.34 \times 10^{7}, 2.363 \times 10^{7}\right], \sigma \in[3300,4400]\right)$ and $\left(a \in\left[2.34 \times 10^{7}, 2.3635 \times 10^{7}\right], \sigma \in[3400,5000]\right)$, respectively. The focus of this research is to investigate the effect of this parameter estimation uncertainty on the prediction of the perforation probability, $F_{\text {sim }_{i}}$ (simulated frequency of perforation). In fact, uncertainty propagation using Monte Carlo simulations can be performed in order to visualize the distributions of $F_{\text {sim }}$ based on the distribution of $a$ and $\sigma$. Therefore, the error in the estimation of the stochastic model parameters is propagated to the model output which lead to the establishment of the numerical distribution. In this implementation, first, the two distribution are going to be compared using the best-found parameters. Afterwards, the parameters are randomly selected from the initially established optimization sample. Finally, $(a, \sigma)$ are uniformly selected from their corresponding 
B. Tahenti et al., Accuracy Analysis of the Brownian Motion Approach for the Ballistic Resistance...

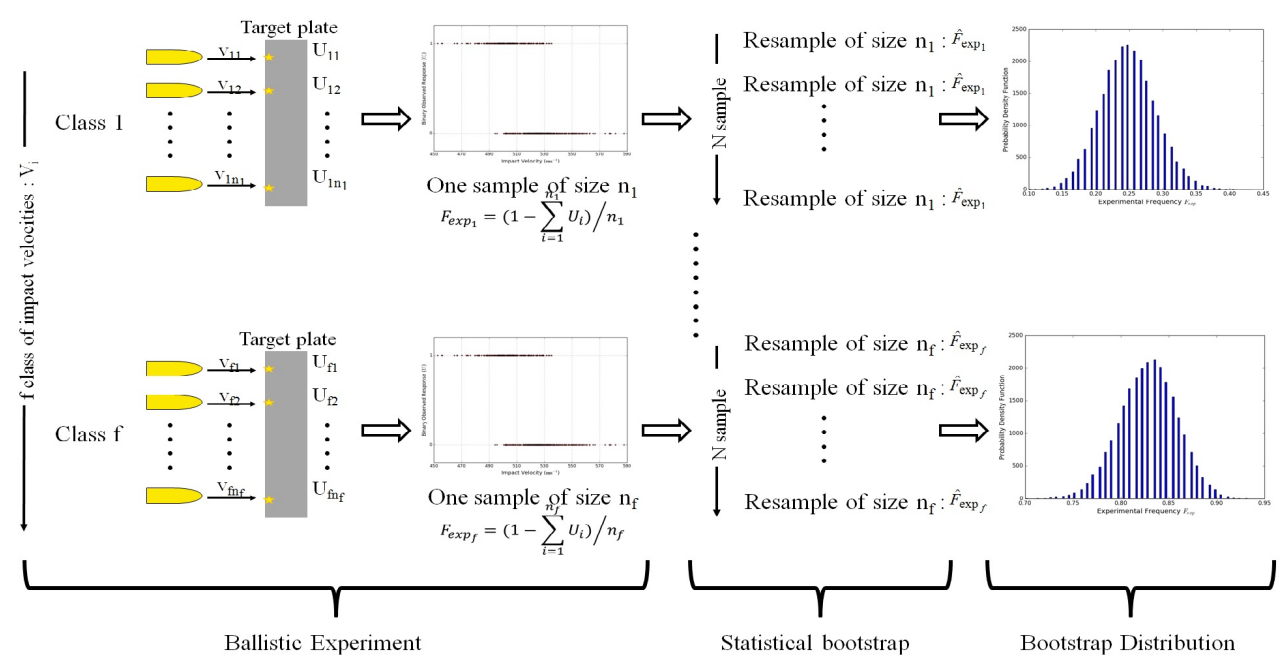

Figure 1. Conceptual draw of the bootstrap method.

intervals. Consequently, to enable a fair comparison of numerical and experimental results, the numerical experiments are executed in the same conditions as the observed ballistic experiments. Thus, for each impact velocity $V_{i}$, the selected couple $(a, \sigma)$ is inserted in the stochastic model defined in equation 1 . The observed response is $U_{i}=0$ if the bullet penetration $\mathrm{X}$ is greater than the target thickness $\mathrm{H}$ (perforation) and $U_{i}=1$ if not (no perforation). In other words, a numerical contingency table is created in the same way as the experimental one. Repeating this operation $\mathrm{N}$ times provides the distribution of the $F_{\text {sim }}$ regarding the uncertainty on the parameters $(a, \sigma)$ using the Brownian motion approach.

\section{RESULTS AND DISCUSSION}

\subsection{Experimental Versus Numerical Distributions}

In order to verify the accuracy of the Brownian motion approach in modelling ballistic experiments, numerical and experimental distributions of the predictions are presented side by side in figure 2 . Further, figure 2 illustrates the obtained results for the classes 2,3,4,5 and 6 of impact velocities (those are the classes that experimentally show a variability in the target response). Statistical bootstrap allowed the establishment of $\hat{F}_{\exp _{i}}$ distribution based on the observed sample per class of impact velocities. The distribution of $F_{\text {sim }_{i}}$ is constructed using the Brownian motion approach based on the parameters $(a, \sigma)$ that give the best fit to experimental results.

Unfortunately, at first glance, it seems that there is a given inconsistency between numerical and experimental distributions especially for low and high probabilities of perforation (class 2 and 6 of extreme probabilities). However, visual inspection of the two distributions in each class shows that they almost have the same shape, whereas, the Brownian motion approach produces larger distribution (therefore smaller amplitude) than the experimental one. In fact, the major discrepancy in the classes 2, 3, 5 and 6 manifests in the bias between the means of the two distributions. It can be seen that this bias increase as impact velocities with low and high probability of perforation are searched (the absolute value of the bias is $0.036,0.022,0.019$ and 0.047 respectively). The most interesting result of this analysis is the excellent agreement between the experimental and numerical distribution in the $4^{\text {th }}$ class. In fact, this class is expected to contain the $V_{50}$ velocity. This results is another evidence in favour of this modelling approach in the presence of the restrictive assumption of the constant deceleration and diffusion in the model. In detail, Tahenti et al. (2017) showed that deceleration prediction was consistent with the average resisting force applied on the bullet as explained by Rosenberg and Dekel (2012). In such conditions, the model appears to catch the mean behaviour of the protection (impact velocities near the $V_{50}$ ) however as impact velocities shrink to extreme values, the model starts to show some discrepancy. Therefore, this fact encourages the attribution of those differences to the model assumption rather than to its inability to represent the randomness of the phenomena. 
B. Tahenti et al., Accuracy Analysis of the Brownian Motion Approach for the Ballistic Resistance...
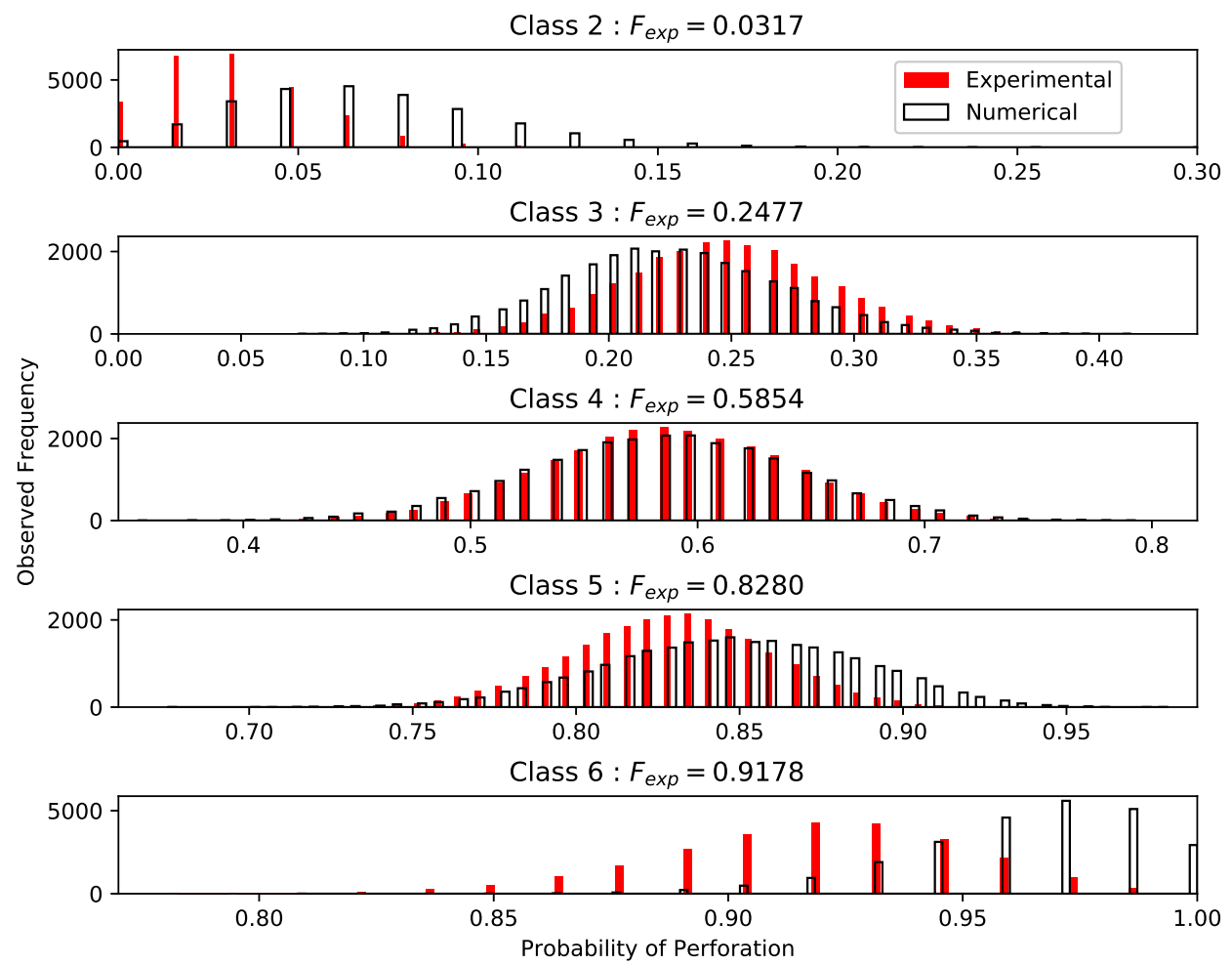

Figure 2. Experimental and numerical histograms of the perforation probability in the classes $2,3,4,5$ and 6 of table 1.

\subsection{Effect of Model Parameters Uncertainty}

After having investigated the behaviour of the two distributions using the best fit results, the uncertainty effect is going to be analyzed. Figure 3 displays the distributions of the $4^{\text {th }}$ class results using the optimization parameters and uniformly selected parameters. It can be seen that the uncertainty in parameters estimation does not cause a remarkable deterioration in the agreement between the experimental and the numerical distributions. In this analysis, the distribution of the results in the classes 2,3,5 and 6 were not shown given that the main observations of the previous section holds without appearance of meaningful differences. This investigation demonstrates that the model does not present a high sensitivity regarding the estimation of its parameters. It is worth remarking that the numerical distribution encompass the variability relative to the stochastic model itself (manifesting in the diffusion coefficient) and the variability in the parameters estimation $(a, \sigma)$. Unfortunately, it is important to note here the stochastic behaviour of the simulations. Therefore, from one simulation to another the differences may become more or less significant.

\subsection{Analysis of the Phenomena Variance}

The statistical bootstrap can provide pertinent information in the interest of understanding the impact velocity effect on the precision of the perforation probability estimation. The standard deviation of the experimental distribution quantify the estimation uncertainty. Figure 4 presents the evolution of the experimental and numerical standard deviation as a function of the bullet impact velocity. The penetration process is governed by the balance between the bullet performances and the target resistance. It is expected that for low impact velocities the target resistance is dominant. Accordingly, the variability in the system response is low. As impact velocity increase, this balance is reached (around the $V_{50}$ velocity). Then, a high dispersion in the system response can be seen. Instead, for high impact velocity the bullet performance increases and consequently the phenomena reaction tends to become more repetitive. This is the physical interpretation of the curve shapes depicted in figure 4 . The numerical model presents the same behaviour as the experimental one with a higher variability which surely include an amount that is inherent to Monte Carlo simulations. Finally, the model reproduce again the experimental observation and unfortunately, a larger experimental sample can allow a better analysis 
B. Tahenti et al., Accuracy Analysis of the Brownian Motion Approach for the Ballistic Resistance...

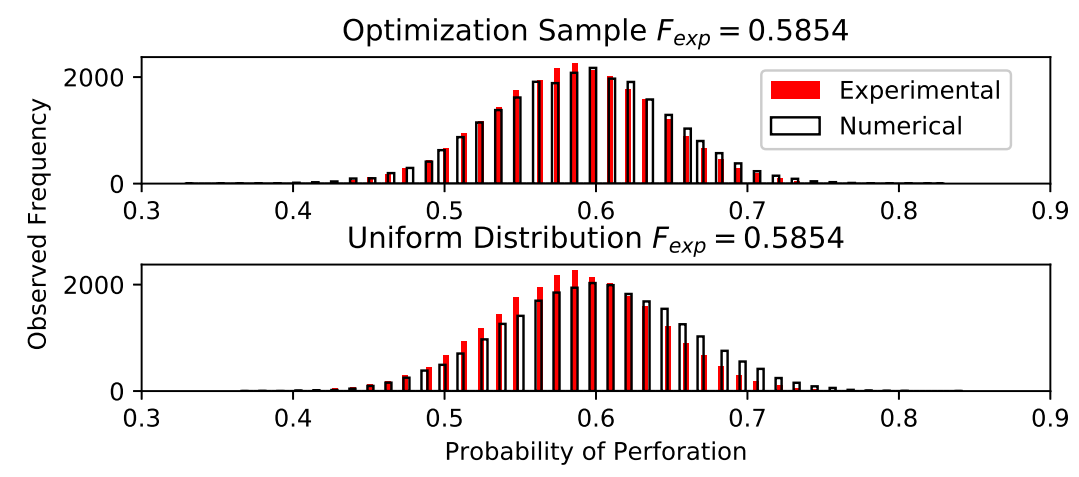

Figure 3. Experimental and numerical histograms of the perforation probability in class 4 of table 1 .

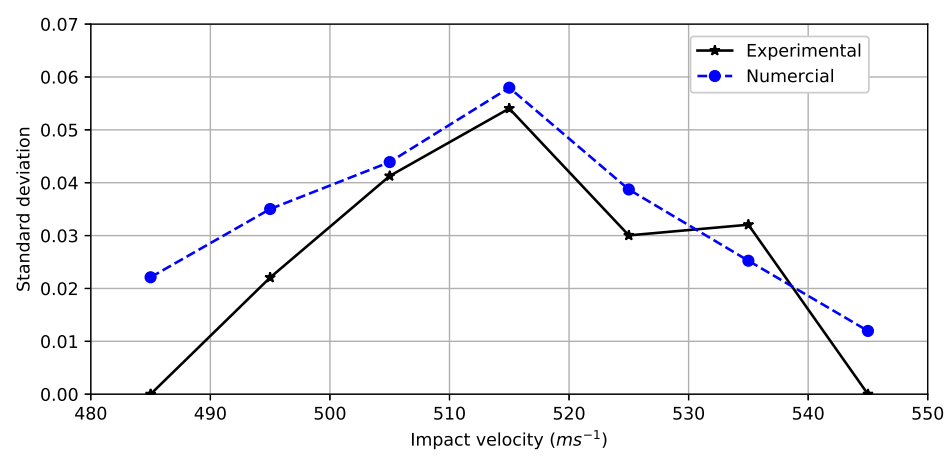

Figure 4. The distribution standard deviation as a function of the bullet impact velocity.

of the model consistency (more confidence is affected to experimental results and the irregular behaviour for $V_{i}=535 \mathrm{~m} \mathrm{~s}^{-1}$ will be attributed to the model weaknesses).

\subsection{Difference in Proportions}

In order to further analysis the accuracy of the Brownian motion approach, the variability of the numerical distribution is studied. In fact, if the experimental distribution is considered as representative of the observed system, the bias of the numerical model can be measured by the difference: $\hat{p}=\bar{F}_{\text {sim }_{i}}-\bar{F}_{\text {exp }_{i}}$ (the bar symbol is used to define the mean of the distribution of the expectations $\hat{F}_{\text {sim }_{i}}$ and $\hat{F}_{\text {exp }_{i}}$ ). It was shown that the two distributions have almost the same shape, hence the variability of the bias is an indicator that reflect the numerical model accuracy regarding the experimental one which is considered as the reference value in this analysis. The models parameters uncertainty is accounted for by uniformly selecting the couple $(a, \sigma)$ from their associated intervals. Figure 5 displays the distribution of $\hat{p}$ based on 100 samples of numerical and ex-

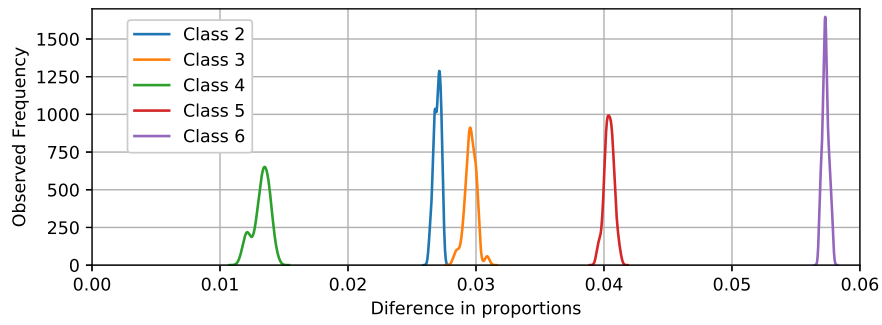

Figure 5. Density function of the differences in proportions of the classes 2,3,4,5 and 6 of table 1. 
B. Tahenti et al., Accuracy Analysis of the Brownian Motion Approach for the Ballistic Resistance...

perimental distribution per class of the fifth observed classes. In addition, table 2 summarize the characteristic statistics of the displayed distributions. it can be seen that the Brownian motion estimation lies on a maximum

Table 2. First and second moments of the difference in proportions.

\begin{tabular}{|c|c|c|c|}
\hline Class & $\mathbf{F}_{\text {exp }}$ & $\operatorname{Mean}(\hat{\mathbf{p}})$ & $\operatorname{Std}(\hat{\mathbf{p}})$ \\
\hline Class 2 & 0.0317 & 0.027 & 0.00027 \\
\hline Class 3 & 0.2477 & -0.03 & 0.00047 \\
\hline Class 4 & 0.5854 & 0.013 & 0.00067 \\
\hline Class 5 & 0.8280 & 0.04 & 0.00037 \\
\hline Class 6 & 0.9178 & 0.057 & 0.00025 \\
\hline
\end{tabular}

distance of $\approx 11 \%$ of the expected real value in the classes $3,4,5$ and 6 . However, in the second class, where the perforation probability is very law, the error is considerably high and equal to $\approx 85 \%$. However, table 2 and figure 5 confirm again the good performance of the model in the central class around the $V_{50}$. This result encourages the fact that the model can reproduce this phenomena. Therefore, the model parameter should be better tuned in the goal of seeking the best accuracy and precision in relation to experimental observations in all the classes of impact velocities.

\section{CONCLUSION}

Ballistic response randomness has been modelled using the Brownian motion approach. Statistical inference tools like Chi-square and Kolmogorov-Smirnov goodness of fit test has been implemented for model parameters estimation. However, this estimation is characterized by a given uncertainty. The actual work covered the analysis of the model accuracy and sensitivity regarding this uncertainty. The inspection of the numerical and experimental distributions of $F_{s i m_{i}}$ and $\hat{F}_{\exp _{i}}$ showed that the model has a low sensitivity to the inference uncertainty. The current study put in evidence the effect of the impact velocity on the model accuracy. Under the assumption of constant parameters (the bullet deceleration and the diffusion coefficient), the stochastic model has an excellent agreement with experimental results around the $V_{50}$ velocity even with uncertainty estimation included. As impact velocities shrink to extreme values some discrepancy start to appear between the numerical and experimental results. Therefore, the main conclusion of this modelling approach is that it has a good ability to reproduce the observed variability. However, more effort should be oriented to consider the effect of the impact velocity in the model. So, the Brownian motion approach can reproduce the observed randomness at any impact velocity.

\section{REFERENCES}

Coghe, F., A. Lenom, B. Lawens, B. Tahenti, M. Maldague, and M. Pirlot (2016). The V50 approach revisited: Application of the brownian motion theory. In 29th International Symposium on Ballistics.

Department of Defense (18 December 1997). MIL-STD-662F standard : V50 ballistic test for armor.

Finney, D, J. and F. Tattersfield (1952). Probit analysis: A statistical treatment of the sigmoid response curve. Journal of the American Statistical Association, 47, 687-691.

Kneubuehl, B. P. (2003). Ballistic protection. Swiss Defense Procurement Agency, Thun.

Langlie, H. (1963). A reliability test method for" one-shot" items. Technical report, DTIC Document.

Maldague, M., F. Coghe, and M. Pirlot (2010). Evaluation of the Gauss Probability Function in case of low (high) values of perforation probability. In Proceedings of the Personal Armour Systems Symposium.

National Institute of Justice (2008). 0101.06. Ballistic Resistance of Body Armor.

NATO, J. (2003). Standardization agreement (STANAG) 2920 ed 2 ballistic test method for personal armor materials and combat clothing.

Rosenberg, Z. and E. Dekel (2012). Terminal ballistics. Springer Science \& Business Media.

Tahenti, B., F. Coghe, R. Nasri, and M. Pirlot (2017). Armors ballistic resistance simulation using stochastic process modeling. International Journal of Impact Engineering 102, 140-146. 